\title{
Heartless, a Drosophila FGF receptor homolog, is essential for cell migration and establishment of several mesodermal lineages
}

\author{
Merav Beiman, Ben-Zion Shilo, ${ }^{1}$ and Talila Volk \\ Department of Molecular Genetics, Weizmann Institute of Science, Rehovot 76100, Israel
}

\begin{abstract}
A Drosophila FGF receptor homolog (DFGF-R2/DFRI) termed Heartless (Htl) is expressed in the embryonic mesoderm. The phenotypes of null mutant embryos demonstrated that $\mathrm{Htl}$ is a central player that is required for the development of several mesodermal lineages. No abnormalities in the primary specification of the mesoderm were observed. The first defects were seen as irregular migration and spreading of the mesoderm over the ectoderm. Subsequently, cell fates were not induced in several lineages including the visceral mesoderm, heart, and the dorsal somatic muscles. The defects in the induction of cell fates are likely to result from failure of the mesoderm to spread over the ectoderm and receive patterning signals. The defective spreading could be circumvented in htl mutant embryos by providing an ectopic Dpp patterning signal, leading to the formation of heart and dorsal muscle cells. Htl appears to be required also subsequently during the migration and morphogenesis of the different lineages. Expression of a dominant-negative htl construct after the initial induction of cell fates gave rise to aberrant migration and organization of the visceral mesoderm, heart, and somatic muscles. Thus, a common role for $\mathrm{Htl}$ in cell migration and tissue organization may account for the pleiotropic defects of the htl mutation.
\end{abstract}

[Key Words: Drosophila; FGF receptor; mesoderm; heart; muscles; cell migration]

Received June 6, 1996; revised version accepted October 14, 1996.

The development of tissues and organs in the Drosophila embryo is initiated by determination of the cells that will become the progenitors of the tissue. Subsequently, discrete cell fates are allocated within the tissue, using spatial information received from neighboring tissues and from cell-cell interactions within the tissue itself. Development of the mesoderm is a case in point; upon subdivision of the ectoderm into discrete domains in the Drosophila embryo, the mesoderm is determined by the nuclear gradient of the maternal protein Dorsal (for review, see St. Johnston and Nüsslein-Volhard 1992). In the ventral part of the embryo, encompassing $\sim 20$ cell rows, the nuclear concentration of Dorsal is maximal and the transcription of the zygotic twist and snail genes is induced (Jiang et al. 1991; Ip et al. 1992). twist encodes a transcription factor of the basic helix-loop-helix region (bHLH) family, and plays a key role in the induction of mesoderm-specific genes (Thisse et al. 1988; Murre et al. 1989). snail encodes a zinc finger protein (Boulay et al. 1987) that functions as a transcriptional repressor, and is responsible for the exclusion of midline and neuroectoderm-specific genes from the mesoderm (Nambu et al. 1990; Leptin 1991; Rao et al. 1991).

${ }^{1}$ Corresponding author.
Following the primary induction of mesodermal fate, the cells invaginate during gastrulation. Subsequently, the cells divide and spread to form the second germ layer over the ectoderm. The mechanism dictating mesoderm cell spreading is unknown. Subdivision of the mesoderm into several lineages, including the visceral mesoderm, heart, somatic muscles, and fat body, takes place after the division and spreading of the mesodermal precursors have been completed. The different fates appear to be dictated by the position of the cells: Dorsal mesodermal cells will form the visceral mesoderm and heart, whereas the ventral cells contacting the neuroectoderm will form the somatic muscles. Segregation of the mesoderm into the different cell fates depends on integration of signals originating from the ectoderm and the mesoderm. The Dpp and Wingless proteins, which are expressed in the ectoderm, are essential for patterning the underlying mesoderm (Staehling-Hampton et al. 1994; Baylies et al. 1995; Frasch 1995; Lawrence et al. 1995; Park et al. 1996). Cell-cell interactions within the mesoderm also play a role in allocation of the correct number of cells to each lineage (Corbin et al. 1991). Transplantation experiments suggest that during gastrulation the mesodermal identity of the cells has been determined already, but the specific cell fate has not been assigned 
yet and depends upon the position of each cell (Beer et al. 1987).

Two FGF receptors have been identified in Drosophila. DFGF-R1 (Breathless, Btl) is expressed in the tracheal system and in the midline cells, and is required for cell migration in both tissues (Klämbt et al. 1992). This work will describe a genetic and functional analysis of the roles of DFGF-R2, the second Drosophila FGF receptor homolog, which maps to position 90E. DFGF-R2 has the typical structure of an FGF receptor, with two immunoglobulin domains in the extracellular region and a split cytoplasmic tyrosine kinase domain (termed DFRl in Shishido et al. 1993). In the embryo, it is first expressed during the cellular blastoderm stage, after the primary fate of the mesoderm has been determined by Twist. Later on, DFGF-R2 is expressed in the visceral mesoderm, heart, and somatic muscles (Shishido et al. 1993; Fig. 1). In view of the expression pattern of DFGF-R2 and the roles assigned to FGF receptors during mesoderm development in other species (see Discussion), it was of interest to determine the function of DFGF-R2 in the development of the mesoderm.

This work shows that DFGF-R2 (termed Heartless on the basis of the mutant phenotype) is a key player in the

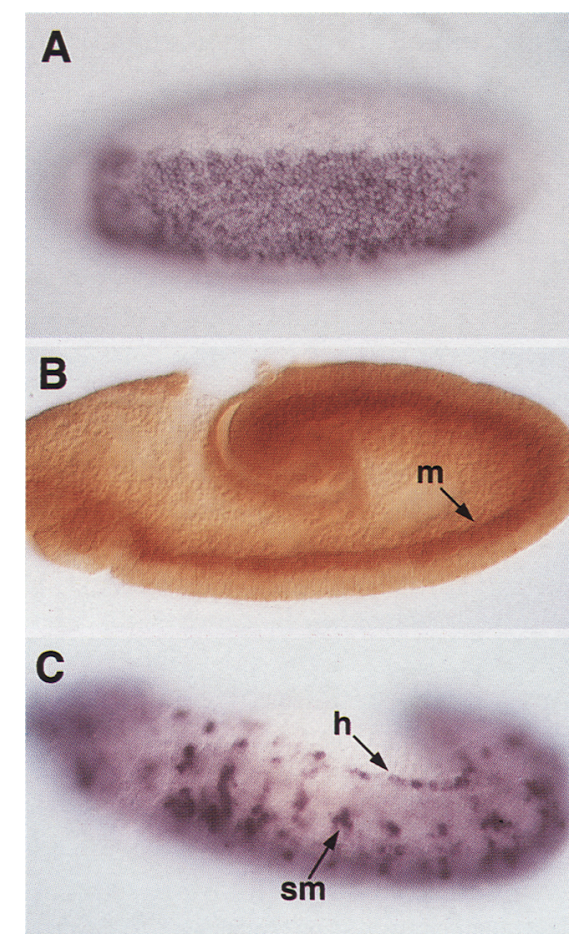

Figure 1. Expression of $h t l$ during embryogenesis. (A) At stage 5 , expression of $h t l$ RNA is observed in a rectangle of cells that will invaginate to form the mesoderm. $(B)$ Following gastrulation, at stage 8 , anti- $\mathrm{Htl}$ antibodies first detect the appearance of the protein in the mesodermal cells $(\mathrm{m})$, which have already invaginated. (C) At stage 12, htl RNA is detected in the heart precursors (h) and the somatic muscle cells $(\mathrm{sm})$. In all figures anterior is to the left. determination of mesodermal cell fates in three lineages: Visceral mesoderm, heart, and somatic muscles. Embryos homozygous for null mutations in the gene do not develop visceral mesoderm and heart tissues. In addition, the somatic muscles are significantly reduced and abnormally arranged. This wide spectrum of Heartless (Htl) functions may result from a common function of the receptor in directing cell migration and tissue organization. The mesoderm cells of $h t l$ mutant embryos do not spread properly to reach the positions where they normally receive patterning cues from the ectoderm. Indeed, some of the defects could be rescued by providing ubiquitously the Dpp patterning signal, which is normally displayed by a restricted set of ectodermal cells. Utilization of a dominant-negative Htl construct demonstrated that for each of these tissues, the role of the receptor is not restricted to the initial establishment of the lineage. Subsequently, $\mathrm{Htl}$ is also required during cell migration and tissue morphogenesis.

\section{Results}

\section{htl expression}

In situ RNA hybridization showed that $h t l$ transcription is restricted to the mesoderm. Expression begins at cellular blastoderm (stage 5), in a ventral rectangle of cells defining the region that will invaginate and form the mesoderm (Fig. 1A). The protein is first seen at stage 8 , after the invagination of the mesoderm (Fig. 1B). The time of appearance of the protein suggests that it does not function in the primary determination of the mesodermal precursors, nor in the process of ventral furrow formation. Indeed, in $h t l$ mutant embryos both of these processes are normal (see below). Expression of $h t l$ in the mesoderm persists during embryogenesis, and is detected in the visceral mesoderm, heart, and somatic muscle precursors (Fig. 1C).

\section{Deficiencies in the htl locus}

The entire coding region of $h t l$ is included within a single exon. A $5^{\prime}$ noncoding exon (exon I) is located in close proximity (Ito et al. 1994). In situ hybridization with exon I- or exon II-specific probes shows identical expression patterns (not shown). The $h t l$ locus is located in close vicinity to the stripe (sr) gene. The $s r^{B 14}$ element inserted in $s r$ is homozygous viable, and shows defects in adult muscle patterning. It was used as a source for local transposition. The resulting lines were screened for lac $\mathrm{Z}$ expression which would mimic the $h t l$ pattern, and one such line (P16) was identified. Southern blotting showed that the element has inserted upstream to $h t l$ exon I, and the original $\mathrm{sr}^{B 14}$ element has been retained (not shown). Although the P16 line was lethal, it did not display any embryonic defects. An imprecise excision strategy was thus utilized. All the lethal lines that were isolated displayed loss of both elements and presumably the sequences between them. Two lines that were further 
characterized $\left(h t l^{\Delta 30}\right.$ and $\left.h t l^{\Delta 51}\right)$ show a loss of $h t l$ exon I but not exon II, and do not express $h t l$ (not shown).

Although these lines are also defective in the sr locus, as a result of the late expression and requirement for $\mathrm{Sr}$, the overlap between the phenotypes resulting from $\mathrm{mu}$ tations in the two genes is likely to be marginal. The $\mathrm{Sr}$ protein is first detected at stage 12 , in the muscle attachment sites on the ectoderm, and is required for correct muscle patterning (Volk and VijayRaghavan 1994; Frommer et al. 1996).

A second approach to generate mutations in htl utilized mobilization of the P1618 element, which is inserted in an intron of $s r$. In one of the derived lines (termed $h t l^{L H 14}$ ), Southern blotting shows that both $h t l$ exons were excised. This line may have undergone a "hit and run" alteration in the vicinity of the $h t l$ locus, with retention of the original element and without suffering any modifications in the $s r$ locus: The $h t l^{L H 14}$ deficiency is viable over $s r^{155}$, the most severe $s r$ allele available.

The excisions described above are much more restricted than the $s r^{16}$ deficiency, which removes in addition to $h t l$, the $s r$ and couch potato genes. They provide DFGF-R2 null alleles and give rise to identical phenotypes. Although the complication of defects in the $s r$ gene should be taken into account, it is possible to define the phenotypes in the visceral mesoderm, heart, and somatic muscles that occur prior to the expression of $s r$, and result specifically from defects in $h t l$ (see below). For most of the phenotypic analyses described below the $h t$ $1^{L H 14} / \mathrm{ht} \mathrm{I}^{\Delta 30}$ heteroallelic combination was used, and gave identical results to homozygous combinations of each deficiency.

As a complementary tool to investigate the roles of $h t l$, an inducible dominant-negative $h t l$ (DN-htl) line was generated. Dominant-negative constructs have proved useful for the analyses of FGF receptor functions in Drosophila, Xenopus, and mice (Amaya et al. 1991; Reichman-Fried and Shilo 1995; Robinson et al. 1995). The construct encodes the normal extracellular and transmembrane domains, but is lacking the cytoplasmic kinase domain. It is regulated by the Gal4 UAS sequence (Brand and Perrimon 1993), and was induced at different times of embryogenesis by the heat shock-Gal4 (hs-Gal4) construct.

\section{The primary defects of htl mutant embryos}

The fate of the mesoderm of $h t l$ mutant embryos was followed by the expression of DMef2, which is expressed early in all mesodermal cells (Lilly et al. 1994; Bour et al. 1995). Although invagination of the mesoderm took place, an irregular spreading and arrangement of the cells was observed. Cross-sections demonstrate that in wildtype, stage 9 embryos [ $4 \mathrm{hr}$ after egg laying (AEL)], spreading of the mesoderm has been completed, and a uniform, single layer of cells extending up to the dorsal ectoderm was formed (Fig. 2A). In contrast, $h t 1$ embryos collected at the same stage show a highly irregular organization of the mesoderm. Multiple layers and clusters of cells are seen, some mesodermal cells are found at aberrant posi-

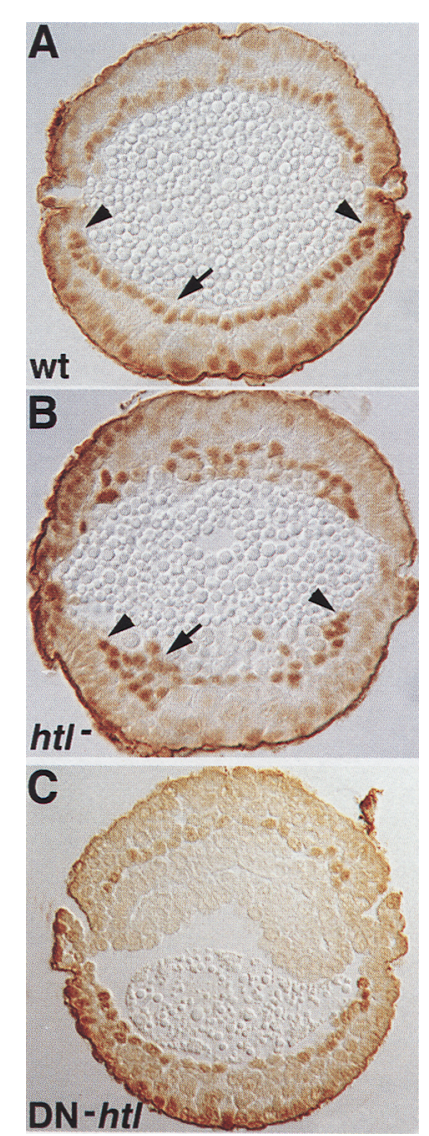

Figure 2. Early defects of htl mutant embryos. $(A)$ Cross-section of a stage 9 wild-type embryo shows the formation of a single layer of mesoderm (arrow) that spreads and contacts both ventral and dorsal ectoderm. $(B)$ Cross-section of a stage 9 $h t l^{\Delta 30} / h t I^{L H 14}$ embryo shows defects in the migration and organization of the mesodermal cells. The arrow shows the accumulation of the mesodermal cells in several layers in the mutant embryos, and the arrowheads show the position of the dorsal-most mesodermal cells in wild-type and mutant embryos. (C) Induction of the DN-htl construct by hs-Gal4 at $2.5 \mathrm{hr}$ AEL does not lead to defects in mesoderm spreading by $4 \mathrm{hr}$ AEL. The mesoderm is labeled with anti-DMef 2 antibodies.

tions, and in general they fail to migrate toward the dorsal ectoderm (Fig. 2B).

\section{The htl visceral mesoderm phenotype}

The visceral mesoderm develops from the dorsal mesodermal cells. It is derived from metamerically repeated primordia of the early mesoderm. Later on, the visceral mesoderm forms a continuous band of cells with no morphological signs of segmentation. The expression pattern of the bagpipe (bap) gene marks the formation of the visceral mesoderm (Azpiazu and Frasch 1993; Fig. 3A,B). Initially, bap is expressed in segmental patches along the anterior-posterior axis. Later, the patches elongate and finally merge with each other, assume a columnar shape and form the continuous band of the visceral mesoderm. When this tissue is fully differentiated, expression of the 
Figure 3. htl visceral mesoderm mutant phenotypes. $(A)$ In wild-type embryos, expression of bap marks the development of the visceral mesoderm. At stage $10 \mathrm{bap}$ is expressed in segmental patches. $(B)$ At stage 11 these patches begin to merge. $(C)$ In a homozygous $h t l^{L H 14}$ mutant embryo, an irregular pattern of patches that fail to fuse is observed. The arrow shows weaker staining in the anterior patches. $(D)$ Expression of FasIII is observed in stage 11 wild-type embryos, after the visceral patches have merged. (vm) Visceral mesoderm. (E) In a $h t l^{\Delta 30} / h t l^{L H 14}$ embryo, only small residual patches of visceral mesoderm expressing FasIII are observed (arrow). $\{E \mid$ In embryos carrying the $h s-$ Gal4 and DN$h t l$ elements that were heat shocked at $2.5 \mathrm{hr} \mathrm{AEL}$, patches of FasIII-expressing cells are formed (arrow), but fail to merge.

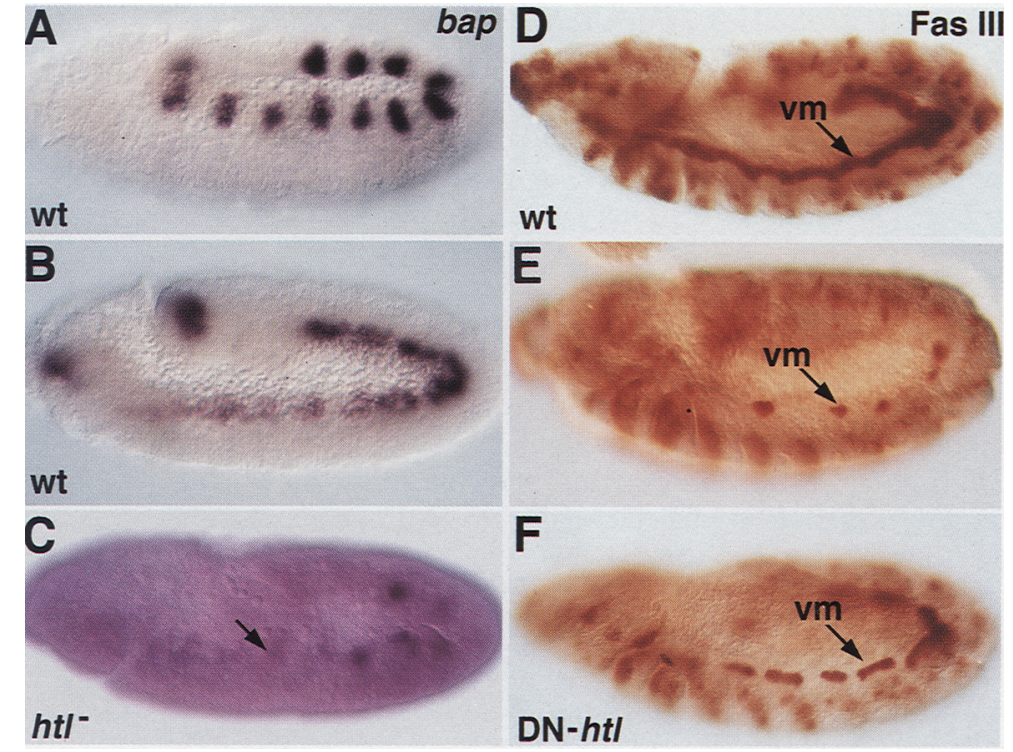

marker FasIII is detected in all of the visceral mesoderm cells as a continuous band (Patel et al. 1987; Fig. 3D).

Upon staining with anti-FasIII antibodies, the null mutant embryos of $h t l$ were lacking the typical staining observed in the visceral mesoderm. Instead, only several very small patches of FasIII-positive cells were monitored (Fig. 4E). When bap was used to follow the fate of the visceral mesoderm in htl mutants, an irregular pattern of expression was observed at the stage when the patches normally fuse (Fig. $3 \mathrm{C}$ ). These results demonstrate that the visceral mesoderm precursors can differentiate in the absence of $h t l$, and the main defect is a significant reduction in the number of cells expressing the markers. In addition, the mutant embryos fail to organize the visceral mesoderm at the stage when migration and fusion of the patches normally take place.

The DN-htl construct provides a complementary approach to dissect the role of $h t l$ in mesoderm development. Typically, a lag period of $1-2 \mathrm{hr}$ is required for the accumulation of the DN-htl construct following Gal4 translation. Induction of the construct at $2.5 \mathrm{hr}$ AEL does not give rise to significant defects in mesoderm spreading, which is normally completed by $4 \mathrm{hr}$ AEL (Fig. 2C). Thus, even early inductions of the DN-htl construct allow analysis of later functions of $h t l$, which are required after the completion of mesoderm spreading.

To study later roles of $h t l$ in visceral mesoderm development, expression of DN-htl was induced by subjecting wild-type embryos to heat-shock $2.5 \mathrm{hr}$ AEL. The treated embryos were fixed at $7 \mathrm{hr}$ AEL and stained with antiFasIII antibodies. Upon heat shock of embryos containing only the $h s$-Gal4 element, mesodermal defects were observed only rarely. The embryos expressing DN-htl exhibit an intermediate phenotype. Instead of a continuous band of FasIII-expressing cells in the visceral me- soderm, only clusters of cells expressing the marker were detected (Fig. 3F). The number of cells expressing FasIII appears normal, but these cells fail to undergo the typical migration leading to the formation of a continuous visceral mesoderm. Induction of DN-htl leads only to an intermediate phenotype, probably as a result of insufficient excess of DN-htl compared with the endogenous receptor. The hypomorphic phenotype of a "broken," unfused visceral mesoderm unravels a second role for the receptor, after the expression of FasIII in the visceral mesoderm has been induced. This function may be involved in the ability of the visceral cells to migrate, merge with cells in the adjacent segments, and form a continuous band. Induction of the DN-htl construct at later times (e.g., at $3.5 \mathrm{hr}$ AEL), did not give rise to any defects in the visceral mesoderm.

\section{htl heart phenotype}

In late stage 11 embryos, the dorsal-most two rows of mesodermal cells split from the visceral mesoderm and give rise to the heart precursors. From stage 12 until after dorsal closure, those cells move dorsally together with the overlying ectoderm until they meet and fuse with the cells migrating from the opposite side. The cells of the dorsal row, which form the dorsal vessel, are contractile, express Myosin, and are termed cardioblasts. The cells in the second row are rounded and give rise to the pericardial cells that flank and support the dorsal vessel (Bate and Rushton 1993; Zaffran et al. 1995).

To determine the role of $h t l$ in heart formation, several markers of developing heart cells were examined. First, the null embryos were stained for early markers [e.g., Even skipped (Eve)], to follow the generation of heart precursor cells. At stage 11, Eve is expressed in a seg- 


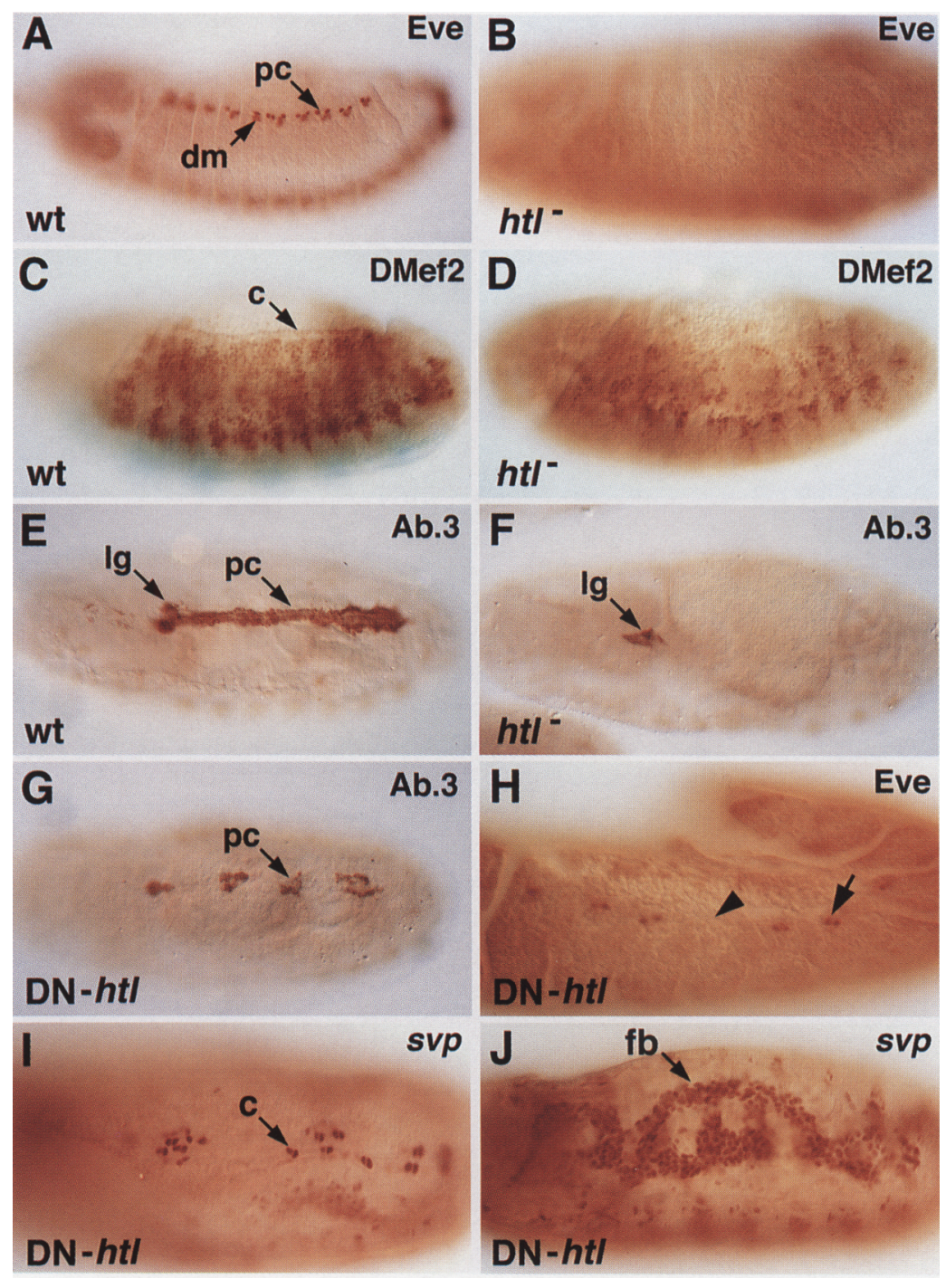

Figure 4. Defects in heart development of $h t I$ mutant embryos. (A) In wild-type embryos the Eve protein is detected at stage 11 in pericardial cells $(\mathrm{pc})$ and dorsal somatic muscles $(\mathrm{dm}) .(B)$ In $s r^{16}$ deficiency embryos, expression of Eve in both lineages is abolished. (C) In wild-type embryos (stage 13) DMef2 is expressed in both cardial heart cells (c) and somatic muscles. $(D)$ In $h t I^{L H 14}$ embryos no expression in the heart is detected, and expression in the somatic muscles is significantly reduced. $(E)$ Antibody \#3 stains the pericardial cells $(\mathrm{pc})$ and the lymph gland $(\mathrm{lg})$. A wild-type embryo at stage 15 is shown. $(F)$ In a $s r^{16}$ embryo, only the staining in the lymph gland is detected. $(G)$ In a $h s$-Gal4/DN-htl embryo induced at $4 \mathrm{hr} A E L$, only patches of pericardial cells are observed. $(H)$ In similar embryos at stage 12, defects in the pattern of Eve expression are observed. In some segments expression is missing altogether (arrowhead), in others it is reduced (arrow). (I) A hs-Gal4/DN-htl embryo containing a $s v p$ enhancer trap was induced at $5 \mathrm{hr} \mathrm{AEL}$, and fixed at stage 15 . Staining with anti- $\beta \mathrm{Gal}$ antibodies shows irregularities in the pattern of the cardial cells $\langle\mathrm{c}\rangle$. $(J)$ The same embryo shows a normal pattern of the fat body $(\mathrm{fb})$. mental pattern in pericardial cells and dorsal somatic muscle precursors (Azpiazu and Frasch 1993; Fig. 4A). In the mutant embryos, no Eve-positive heart precursors or dorsal somatic muscles could be detected (Fig. 4B), whereas the expression in the central nervous system was unaltered.

Because Eve serves as a marker for only a subset of the dorsal vessel precursor cells, we also wanted to examine a gene that is expressed in the cardioblast precursor cells. The DMef2 protein is expressed in all myogenic lineages throughout embryogenesis. During germ band retraction and in older embryos, DMef2 persists in the somatic, visceral, and pharyngeal musculature, as well as in cardial cells (Lilly et al. 1994; Bour et al. 1995; Fig. 4C). In htl mutant embryos, no expression of DMef2 in the cardioblast cells was detected (Fig. 4D).

Finally, a monoclonal antibody termed $\mathrm{Ab}$. \#3 stains all pericardial cells from stage 14, prior to the completion of dorsal closure (Yarnitzky and Volk 1995; Fig. 4E). In the mutant embryos no staining was observed, except for the lymph gland cells located at the anterior part of the dorsal vessel, which are also recognized by this antibody (Fig. 4F). The absence of the cardial cells can also be seen by anti-Myosin staining (not shown). Because absence of the heart represents the most pronounced and defined phenotype of the DFGF-R2 mutants, we term the locus heartless.

The role of $h t l$ in dorsal vessel development was also analyzed by the DN-htl construct. DN-htl was induced at $3 \mathrm{hr} A E L$, and the embryos were fixed at $13 \mathrm{hr}$. Dorsal closure was properly completed, but defects in the organization of both rows of the dorsal vessel were observed. A reduction in the number of pericardial and cardial cells that were formed was observed by staining with $\mathrm{Ab}$. \#3, anti-Eve (Fig. 4G, H), and anti-Myosin antibodies (Fig. 5D). Similar defects were observed when the DN-htl construct was induced up to $5 \mathrm{hr}$ AEL. In this case, the fate of the cells was followed with a seven-up (svp) enhancer trap. Whereas the formation of the fat body appears normal, the cardial cells are reduced in number 
Figure 5. htl somatic muscle mutant phenotypes. $(A)$ Wild-type embryo at stage 16 stained with anti-Myosin. $(B)$ In hsGal4/DN-htl embryos (induced at $4 \mathrm{hr}$ AEL), missing ventral muscles (arrow) or misdirected muscles (arrowhead) are seen. (C) In $h t l^{\Delta 30} / h t 1^{L H 14}$ embryos only a smaller number of muscles is stained. These muscles show irregular organization and attachment. $(D)$ In a similar embryo a dorsal view shows missing muscles (arrow), unfused round muscle cells (arrowhead), and residual cardial cells (c) expressing Myosin.

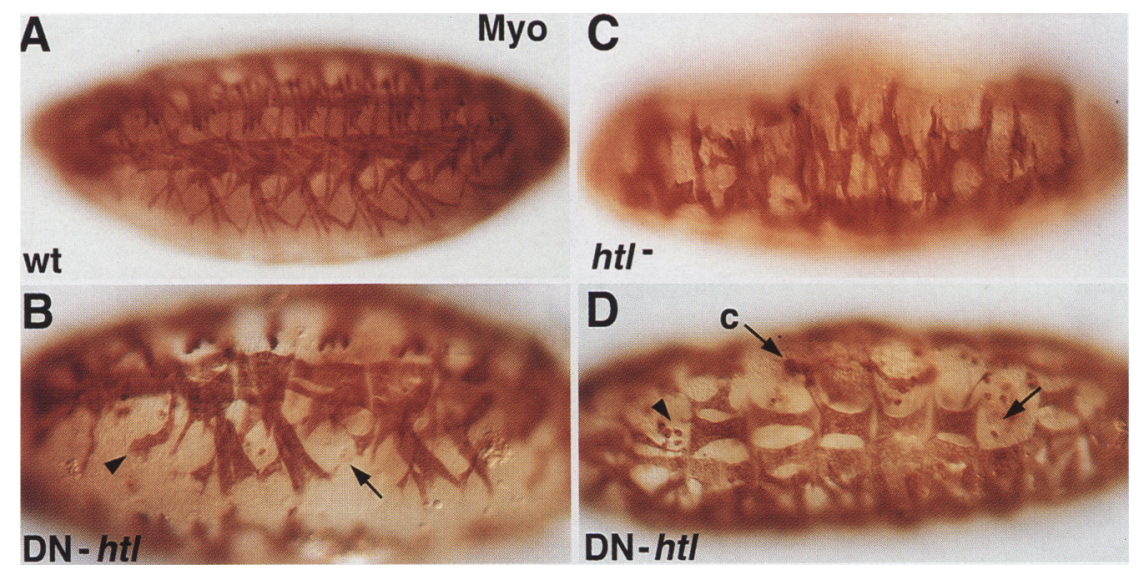

(Fig. 4I,J). Given the lag period required to induce the accumulation of the DN-htl construct, the defects in the formation of the heart cells should be attributed to later functions of the receptor in the migration of the dorsal vessel cells or in the organization of this tissue.

\section{htl somatic mesoderm phenotype}

The ventral-most portion of the mesoderm gives rise to the somatic musculature. This tissue forms the complex pattern of muscles on the inner surface of the epidermis (Bate 1990). At the onset of germ band retraction, somatic founder cells expressing distinct markers (e.g., Eve, Kr, or Nautilus) are determined. During germ band retraction, cells of the ventral-most mesoderm begin to fuse with the founder cells, to form the different muscle types. By the end of germ band retraction, most of the 30 muscle types of every segment are represented by a syncytial precursor at a specific site in the somatic mesoderm. The syncytia fuse with neighboring myoblasts and enlarge. The myotubes send out growth cone-like processes that migrate over the epidermal surface to the various sites where muscle attachments will form. By 13 hr AEL, muscle morphogenesis is completed (Bate and Rushton 1993).
To determine the role of $\mathrm{Htl}$ in the formation of the somatic muscles, the phenotype of null mutant embryos was examined by antibodies to DMef2, Eve, and Myosin. The somatic expression of DMef2 is detected in segmentally repeating clusters of muscle progenitors (Lilly et al. 1994; Bour et al. 1995; Fig. 4C). htl null mutant embryos exhibit extensive reduction in the number of DMef2expressing cells at stages $12-14$, particularly in the lateral and dorsal region of the embryo (Fig. 4D). Eve staining in the dorsal muscles is also missing (Fig. 4B).

The body wall muscles, which are arranged in ventral, pleural, and dorsal groups, are visualized by anti-Myosin staining (Fig. 5A). This antibody also stains the central tube of cardial cells extending along the dorsal midline. In $h t l$ null mutant embryos, only residual somatic muscles that retained Myosin expression were observed. As was predicted from the Dmef2 and Eve staining, fewer muscles were formed. This reduction has also been demonstrated by following the expression of nautilus (Shishido et al. 1993). Even in the cells that did express Myosin, severe defects were observed (e.g., the muscles exhibited abnormalities and irregularities in patterning and attachment to the ectoderm; Fig. 5C). In addition, a significant number of round, unfused myoblasts was detected. The observation that dorsal and lat-
Figure 6. Ectopic Dpp circumvents $h t I$ defects. $(A)$ Induction of $d p p$ expression by twi-Gal4 in wildtype embryos gives rise to enlarged clusters of dorsal muscles expressing Eve (arrow). Induction by 69BGal4 gave similar results (not shown). $(B)$ Homozygous $h t t^{\Delta 30}$ embryos do not express Eve in the mesoderm (Fig. 4B). Induction of $d p p$ by $t w i-G a l 4$ in homozygous $h t l^{\Delta 30}$ embryos rescued the defect and gave rise to clusters of pericardial and dorsal muscle cells expressing Eve (arrow). In addition, aberrant lateral clusters expressing Eve were detected (arrowhead). (C) Similar results were obtained when $d p p$ was induced by $69 \mathrm{~B}-\mathrm{Gal} 4$ in homozygous $h t 1^{\Delta 30} \mathrm{em}-$ bryos. $(D)$ Induction of $d p p$ by ptc-Gal4 in homozygous $h t l^{\Delta 30}$ embryos gave rise to a partial rescue of Eve-expressing clusters (arrow).

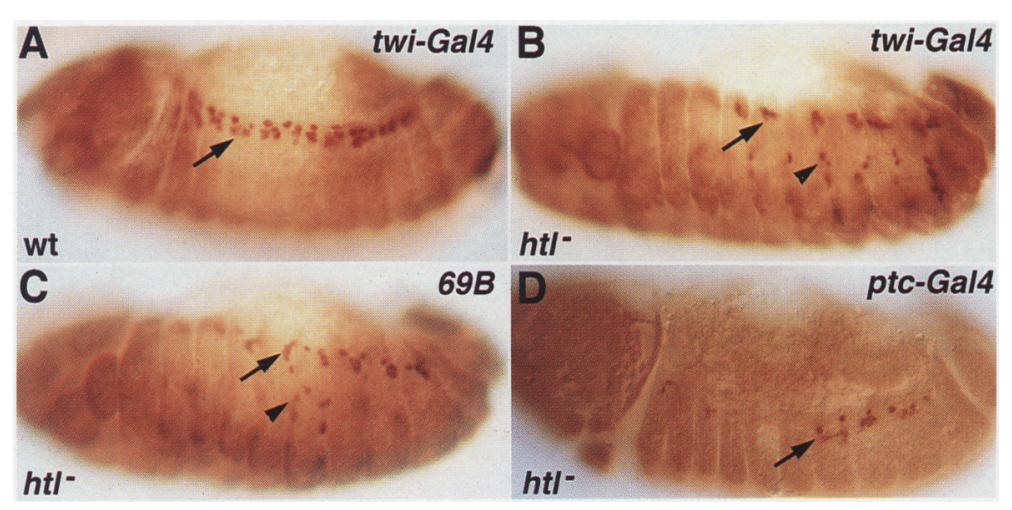


eral somatic precursor cells were not generated in the absence of $h t l$ indicates that it is also crucial for cell fate determination in the somatic mesoderm. The residual Myosin staining, which demonstrated severe defects in muscle patterning, may imply that $h t l$ is needed also for tissue morphogenesis.

The DN-htl construct was used to determine whether $\mathrm{Htl}$ is required continuously during the process of somatic muscle development, or only for its onset. Expression of DN-htl was induced at 3, 4, 5, or $6 \mathrm{hr}$ AEL. The treated embryos were fixed at $13 \mathrm{hr}$ AEL and stained with anti-Myosin antibodies. Severe defects in muscle patterning were observed when the construct was induced at 3-4 hr, and intermediate defects upon induction at 5-6 hr (Fig. 5B,D). Although most of the muscle precursors were formed, as was monitored by anti-Eve or anti-DMef2 staining (not shown), they did not migrate and spread normally, and some remained unfused. These results suggest that $\mathrm{Htl}$ is essential throughout somatic mesoderm development and morphogenesis. Because induction of the dominant-negative construct after $6 \mathrm{hr}$ was not effective, $\mathrm{Htl}$ seems to be essential for the formation of the somatic muscles only up to stage 12 (taking into account a 1-2 hour period required for accumulation of Gal4 and subsequently of DN-Htl). This suggests that $h t l$ is required for the migration of the muscle precursor cells, but not for the attachment of the muscles to the ectoderm, which begins at stage $14(10-11 \mathrm{hr}$ AEL).

\section{Ectopic Dpp circumvents htl defects}

The defects in the induction of cell fates in $h t l$ mutant embryos may arise from an intrinsic defect in the capacity of the mesodermal cells to respond to diverse inductive cues. Alternatively, the early abnormalities in the migration and spreading of the mesoderm in htl mutant embryos may lead to inability of the cells to receive the normal patterning signals from the ectoderm (e.g., Dpp), and, consequently, it may lead to defects in the induction of different cell fates. To distinguish between these possibilities, ectopic expression of Dpp was induced in htl mutant embryos. In wild-type embryos, ectopic Dpp gives rise to an excess of heart and visceral muscle cells (Staehling-Hampton et al. 1994; Frasch 1995; Fig. 6A). If the absence of $h t l$ does not give rise to an intrinsic defect in the response to the inductive signals, we would expect to observe partial rescue of the htl mutant phenotype following ectopic expression of Dpp.

To circumvent the requirements for inductive interactions between the ectoderm and mesoderm, Dpp was ectopically induced in the mesoderm by twi-Gal4, and the pattern of Eve-expressing cells was followed. In wildtype embryos, larger dorsal muscle clusters of Eve-expressing cells are observed, but only in the normal location (Fig. 6A). In the $h t 1$ mutants expression of Eve in the mesoderm was restored by twi-Gal4/UAS- $d p p$. In addition to the clusters of stained cells in the normal dorsal location, lateral clusters were observed (Fig. 6B). A similar result was obtained when the ectopic Dpp was in- duced uniformly in the ectoderm by the 69B Gal4 line (Fig. 6C). Thus, the $h t l$ mesodermal cells retain not only the capacity to respond to Dpp that is displayed by the mesoderm, but also to respond to the ligand secreted by the ectoderm. The induction of lateral clusters in $h t l$ mutant embryos but not in wild-type embryos may result from the early spreading defect or from the presence of a larger number of cells that have not received an inductive cue prior to the ectopic presentation of Dpp. Finally, a more limited rescue of the $h t l$ phenotype is observed when Dpp is ectopically expressed by a restricted set of ectodermal cells following induction by ptc-Gal4 (Fig. 6D). These results strongly suggest that the failure to induce the different mesodermal cell fates in htl mutant embryos is a result of the inability of the cells to migrate to the proper positions, where signals from the ectoderm are responsible for patterning the mesoderm.

\section{Discussion}

\section{The htl phenotype}

Restricted deficiencies and a dominant-negative construct were used to define the mutant phenotype for the DFGF-R2 locus, which we term heartless. The hallmark of the $h t l$ phenotype is its pleiotropy: Several mesodermal lineages, including the visceral mesoderm, heart, and somatic muscles, are severely deranged or missing. The primary specification of mesodermal cells in the embryo, however, is normal in the absence of Htl. This is in accordance with the appearance of the $\mathrm{Htl}$ protein only after invagination of the mesodermal cells, and the dependence of $h t l$ transcription on Twist (Shishido et al. 1993). The first defects are observed at late stage 8 (3-4 hr AEL), when the mesodermal cells spread over the ectoderm. Staining with a tinman probe (not shown) or DMef2 antibodies shows that the cells do not form a uniform layer that is tightly associated with the ectoderm (Fig. 2B). Variable extents of spreading are observed in different parts of the embryo. The early migration defect is likely to play a central role in the abnormalities observed in determination of the fate of the different lineages. This notion is especially pertinent in view of the fact that the mesodermal cells are likely to receive different cues from the ectodermal cells, that are instrumental in the determination of their identity. Indeed, it was possible to rescue the $h t l$ defects in cell fate determination by providing Dpp ectopically to the mesodermal cells that did not reach the location where they should be exposed to the normal Dpp signal (Fig. 6).

In addition to the effect of $h t l$ mutations on several lineages, another hallmark of the mutation is that in every lineage the protein appears to be required multiple times, to carry out diverse functions. Absence of $\mathrm{Htl}$ leads to loss of cell fates: Visceral mesodermal cells fail to express certain markers (e.g., FasIII), and heart precursors as well as dorsal muscles are not established. Later on, after the proper cell identity has been determined, $\mathrm{Htl}$ is required for tissue morphogenesis (including the 
migration of the cells and formation of appropriate contacts with neighboring mesodermal or ectodermal cells). The DN-htl construct is able to block fusion of visceral patches expressing FasIII, and to disrupt the fusion and migration of the somatic muscles.

It is important to note that not all mesodermal lineages appear to require the activity of $\mathrm{Htl}$. The fat body develops from clusters of mesodermal cells that are established at stage 11 , fuse at stage 14 , and migrate to form the pattern of the fat body at stage 15 (Hoshizaki et al. 1994). The expression of $s v p$ was used to follow the fate of the cardial cells and fat body. Induction of DN-htl at 4 or $5 \mathrm{hr} A E L$, prior to the establishment of the fat body fates, gave rise to the typical reduction in the number of cardial cells (Fig. 4I), but did not alter the development of the fat body (Fig. 4J).

The most plausible scenario to account for the pleiotropic effects of the $h t l$ mutation is that the receptor is essential for the ability of the cells to migrate and generate the proper tissue morphology. After induction of the different mesodermal lineages, cell migration is also required for the morphogenesis of each tissue: Patches of visceral mesodermal precursors migrate and form a continuous layer, the heart precursors migrate dorsally and fuse to form the dorsal vessel, and the muscle precursors migrate toward their attachment sites on the ectoderm. Although the cues for each of these migration events are not known, it is reasonable to assume that the ectoderm plays a cardinal role. In the absence of the receptor these defects are manifested not only in the initial spreading of the mesoderm, but also in later events of mesodermal cell migration. The possibility that the $\mathrm{Htl}$ ligand would be expressed on the ectoderm in dynamic patterns that may prefigure the future migration of the mesodermal cells is a very appealing one.

\section{$\mathrm{Htl}$ and $\mathrm{BtI}$}

It is interesting to compare the activities of the two FGF receptor homologs identified in Drosophila, $\mathrm{Htl}$ and Btl, as ascertained from their mutant phenotypes. The two receptors show nonoverlapping patterns of expression in the embryo: $\mathrm{Htl}$ is restricted to the mesoderm, whereas $\mathrm{Btl}$ is expressed in the tracheal system, the salivary duct, and the midline cells (Klämbt et al. 1992; Glazer and Shilo 1991). The nonoverlapping expression and activities of the two receptors also appear to reflect the existence of nonoverlapping ligands. Although the ligands have not been identified to date, dominant-negative constructs of the receptors can be used to test their putative binding activities. A dominant-negative Btl construct expressed in all embryonic cells did not give rise to any defects in mesoderm development (Reichman-Fried and Shilo 1995), whereas the DN-htl construct did not affect tracheal migration (not shown). The ability of the Torsodominant DFGF-R2 chimera to partially rescue the tracheal migration defects of $b t l$ mutant embryos demonstrates that some of the cytoplasmic signaling components (including, e.g., Ras and Raf) are shared between the two receptors (Reichman-Fried et al. 1994).
$\mathrm{Btl}$ is not required for the primary specification of tracheal cell fates. Similar to Htl in the mesoderm, Btl is essential for the migration of the cells in the different lineages in which it is expressed, including the tracheal, midline, and border cells (Klämbt et al. 1992; Murphy et al. 1995|. In addition, Btl is also required for the ability of the cells to alter their morphology and cytoskeletal organization. The capacity of the terminal tracheal cells to send long cytoplasmic extensions forming the tracheoles depends on a functional Btl protein (Reichman-Fried and Shilo 1995). It thus appears that the two FGF receptors are essential for cell migration in the tissues in which they are expressed. Defects in cell fates that are observed in $b t l$ or $h t l$ mutant embryos represent secondary consequences of the initial migration defects.

Corollaries to the roles of FGF receptors in mesoderm development of other species

In Xenopus embryos, a role for the FGF signaling pathway in mesoderm induction has been demonstrated. Injection of a dominant-negative FGF receptor construct into wild-type embryos frequently results in tadpole stage animals displaying nearly complete head differentiation, but lacking trunk and tail structures. These experiments indicated that FGF signaling is involved in specifying the differentiation of ventral and posterior mesoderm in Xenopus (Amaya et al. 1991). Further analysis has demonstrated that the dominant-negative FGF receptor can also disrupt the migration of the dorsal mesoderm, which may affect the induction of target genes such as Xbra (Isaacs et al. 1994).

In mice lacking the fgfr-1 locus, the primary specification of mesodermal cell fates takes place. In addition, ES cells of the same genotype were capable of forming different mesodermal subtypes in teratomas (Deng et al. 1994). However, mesodermal patterning was aberrant in the $f g f r-1$ deficient mice, somites were never generated, and paraxial mesoderm was reduced (Yamaguchi et al. 1994).

A role for FGF signaling in cell migration is supported by the finding that mutations in a C. elegans FGF receptor homolog (Egl-15) block the migration of the sex myoblast cells (DeVore et al. 1995). Egl-15 is not required for the actual motility of the sex myoblasts (SMs), but rather for the normal guidance of SM migration: SMs in egl-15 mutants often do not migrate to their proper positions.

\section{Concluding remarks}

The analyses of mutant phenotypes in the htl locus, encoding a Drosophila FGF receptor homolog, demonstrate that $h t l$ is a cardinal element in the development of several mesodermal lineages. Furthermore, within each of these lineages, absence of $\mathrm{Htl}$ leads to multiple defects in the induction of cell fate and tissue morphogenesis. Common defects in mesodermal cell migration and organization may account for a mechanism by which such diverse processes are regulated by a single receptor. $\mathrm{Fu}$ - 
ture work to identify the $\mathrm{Htl}$ ligands and their expression pattern may shed light on this issue.

\section{Materials and methods}

\section{Plasmid constructs}

The htl genomic region was isolated by low stringency hybridization with a probe containing the DFGF-Rl (btl) fragment encoding the kinase domain. The genomic $h t l$ clones were used to isolate cDNA clones. For in situ hybridization, a probe specific for exon I of $h t l$ was generated as a genomic $0.9-\mathrm{kb}$ HindIII$X m n I$ fragment. A probe specific for the coding exon was generated as a genomic $1.2-\mathrm{kb}$ SalI-BamHI fragment.

To generate the DN- $h t l$ construct, an artificial EcoRI site was generated by PCR, 40 bp upstream to the AUG codon. A T7 tag (Novagene) followed by a termination codon and an $\mathrm{XbaI}$ site, was introduced by an oligonucleotide /using a PCR-generated MluI site) following codon 334, 16 amino acids after the transmembrane domain. The truncated htl construct was cloned into the EcoRI-XbaI sites of pUAST (Brand and Perrimon 1993).

\section{Fly lines}

A transgenic line with an insertion of the DN-htl construct on the second chromosome was obtained. This line is homozygous for the chromosome carrying the construct. To induce expression of the construct, the line was crossed to the K25 line lobtained from E. Hafen, University of Zurich, Switzerland) containing the sev HS-Gal4 construct on the third chromosome. Synchronized embryo collections $( \pm 0.5 \mathrm{hr})$ were grown at $25^{\circ} \mathrm{C}$, heat shocked at $37^{\circ} \mathrm{C}$ for $20 \mathrm{~min}$, and returned to $29^{\circ} \mathrm{C}$.

The $s r^{B 14}$ line (also termed AK1) is a homozygous insertion of P-lwB element (obtained from A. Kolodkin, University of California, Berkeleyl. The $s r^{P 1618}$ is also a homozygous insertion. The $s r^{16}$ is a deficiency encompassing the 90D-E region, removing the genes couch potato, sr, and $h t l$ (Bellen et al. 1992). Local transpositions of the $s r^{B 14}$ element were induced by standard procedures, and the P16 insertion upstream to the $h t l$ promoter was identified by the expression pattern of lacZ. Imprecise excisions of P16 and $s r^{P 1618}$ were induced by standard protocols. The 7842 line fobtained from M. Mlodzik, European Molecular Biology Laboratory, Heidelberg, Germany) is an insertion in the svp locus, which is expressed (among other tissues) in the nuclei of the cardial precursors and fat body. For the ectopic expression of Dpp, a UAS- $d p p$ element on the second chromosome lobtained from M. Hoffmann, University of Wisconsin, Madison) was induced in a $h t I^{\Delta 30}$ homozygous embryo. The inducer lines were $t w i-G a l 4$ on the second chromosome lobtained from $M$. Akam, Wellcome Institute, Cambridge, UK), 69B on the third chromosome (obtained from A. Brand, Wellcome Institute), and ptc-Gal4 on the second chromosome lobtained from I. CamposOrtega, University of Köln, Germany).

\section{Antibodies, probes, and staining procedures}

Antibodies recognizing $\mathrm{Htl}$ were generated in guinea pigs by immunization against the entire extracellular domain, expressed in pATH1. bap probe was obtained from M. Frasch (Mount Sinai School of Medicine, New York, NY), monoclonal antibodies against FasIII were used, rabbit anti-Eve antibodies were obtained from $M$. Frasch, rabbit anti-DMef2 antibodies were obtained from H. Nguyen (Albert Einstein College of Medicine, Bronx, NY), monoclonal antibody \#3 was described previously (Yarnitzky and Volk 1995), and rabbit anti-Myosin antibodies were obtained from P. Fisher (State University of New
York, Stony Brook). Rabbit anti- $\beta$-Gal antibodies (Cappel) and HRP-conjugated secondary antibodies (Jackson) were used.

For sections, embryos were stained with anti-DMef2 as a whole mount preparation, dehydrated, washed in ethanol, and infiltrated with JB-4 embedding media (Polysciences, Inc.), according to the manufacturer's instructions. Embryos were oriented in molds and the resin was allowed to harden in a desiccator. Sections (3-4 mm wide) were obtained with a Sorvall MT2B microtome.

\section{Acknowledgments}

We thank the following colleagues for plasmids and fly stocks: M. Akam, H. Bellen, A. Brand, J. Campos-Ortega, P. Fisher, M. Frasch, E. Hafen, M. Hoffmann, A. Kolodkin, M. Mlodzik, H. Nguyen, and N. Perrimon. We are grateful to Lilly Glazer for carrying out the initial cloning and molecular characterization of $h t l$, to Giora Pasca for generating the $h t l^{L H 14}$ excision, and to A. Michelson for sharing unpublished information. This work was supported by grants from The German-Israeli Foundation, the Israel Cancer Research Fund and the Forchheimer Center to B.S., and by a grant from the Israel Cancer Research Fund to T.V.

The publication costs of this article were defrayed in part by payment of page charges. This article must therefore be hereby marked "advertisement" in accordance with 18 USC section 1734 solely to indicate this fact.

\section{References}

Amaya, E., T.J. Musci, and M.W. Kirschner. 1991. Expression of a dominant negative mutant of the FGF receptor disrupts mesoderm formation in Xenopus embryos. Cell 66: 257270.

Azpiazu, N. and M. Frasch. 1993. tinman and bagpipe: Two homeo box genes that determine cell fates in the dorsal mesoderm of Drosophila. Genes \& Dev. 7: 1325-1340.

Bate, M. 1990. The embryonic development of larval muscles in Drosophila. Development 110: 791-804.

Bate, M. and E. Rushton. 1993. Myogenesis and muscle patterning in Drosophila. C.R. Acad. Sci. III 316: 1047-1061.

Baylies, M.K., A. Martinez-Arias, and M. Bate. 1995. wingless is required for the formation of a subset of muscle founder cells during Drosophila embryogenesis. Development 121: 38293837.

Beer, J., G. Technau, and J. Campos-Ortega. 1987. Lineage analysis of transplanted individual cells in embryos of Drosophila melanogaster. IV. Commitment and proliferative capabilities of individual mesodermal cells. Roux's Arch. Dev. Biol. 196: 222-230.

Bellen, H.J., H. Vaessin, E. Bier, A. Kolodkin, D. D'Evelyn, S. Kooyer, and Y.N. Jan. 1992. The Drosophila couch potato gene: An essential gene required for normal adult behavior. Genetics 131: 365-375.

Boulay, J.L., C. Dennefeld, and A. Alberga. 1987. The Drosophila developmental gene snail encodes a protein with nucleic acid binding fingers. Nature 330: 395-398.

Bour, B.A., M.A. O'Brien, W.L. Lockwood, E.S. Goldstein, R. Bodmer, P.H. Taghert, S.M. Abmayr, and H.T. Nguyen. 1995. Drosophila MEF2, a transcription factor that is essential for myogenesis. Genes \& Dev. 9: 730-741.

Brand, A.H. and N. Perrimon. 1993. Targeted gene expression as a means of altering cell fates and generating dominant phenotypes. Development 118: 401-415.

Corbin, V., A.M. Michelson, S.M. Abmayr, V. Neel, E. Alcamo, T. Maniatis, and M.W. Young. 1991. A role for the Droso- 
phila neurogenic genes in mesoderm differentiation. Cell 67: $311-323$

Deng, C.-X., A. Wynshaw-Boris, M.M. Shen, C. Daugherty, D.M. Ornitz, and P. Leder. 1994. Murine FGFR-1 is required for early postimplantation growth and axial organization. Genes \& Dev. 8: 3045-3057.

DeVore, D.L., H.R. Horovitz, and M.J. Stern. 1995. An FGF receptor signaling pathway is required for the normal cell $\mathrm{mi}$ grations of the sex myoblasts in C. elegans hermaphrodites. Cell 83: 611-620.

Frasch, M. 1995. Induction of visceral and cardiac mesoderm by ectodermal Dpp in the early Drosophila embryo. Nature 374: 464-467.

Frommer, G., G. Vorbruggen, G. Pasca, H. Jackle, and T. Volk. 1996. Epidermal egr-like zinc finger protein of Drosophila participates in myotube guidance. $E M B O ~ J$. 15: 1642-1649.

Glazer, L. and B.-Z. Shilo. 1991. The Drosophila FGF receptor homolog is expressed in the embryonic tracheal system and appears to be required for directed tracheal cell extension. Genes \& Dev. 5: 697-705.

Hoshizaki, D.K., T. Blackburn, C. Price, M. Ghosh, K. Miles, M. Ragucci, and R. Sweis. 1994. Embryonic fat-cell lineage in Drosophila melanogaster. Development 120: 2489-2499.

Ip, Y.T., R.E. Park, D. Kosman, K. Yazdanbakhsh, and M. Levine. 1992. dorsal-twist interactions establish snail expression in the presumptive mesoderm of the Drosophila embryo. Genes \& Dev. 6: 1518-1530.

Isaacs, H.V., M.E. Pownall, and J.M. Slack. 1994. eFGF regulates Xbra expression during Xenopus gastrulation. EMBO $J$. 13: 4469-4481

Ito, M., T. Matsui, T. Taniguchi, and K. Chihara. 1994. Alternative splicing generates two distinct transcripts for the Drosophila melanogaster fibroblast growth factor receptor homolog. Gene 139: 215-218.

Jiang, J., D. Kosman, Y.T. Ip, and M. Levine. 1991. The dorsal morphogen gradient regulates the mesoderm determinant twist in early Drosophila embryos. Genes \& Dev. 5: 18811891.

Klämbt, C., L. Glazer, and B.-Z. Shilo. 1992. breathless, a Drosophila FGF receptor homolog, is essential for migration of tracheal and specific midline glial cellls. Genes \& Dev. 6: $1668-1678$.

Lawrence, P.A., R. Bodmer, and J.P. Vincent. 1995. Segmental patterning of heart precursors in Drosophila. Development 121: 4303-4308.

Leptin, M. 1991. twist and snail as positive and negative regulators during Drosophila mesoderm development. Genes \& Dev. 5: 1568-1576.

Lilly, B., S. Galewsky, A.B. Firulli, R.A. Schulz, and E.N. Olson. 1994. D-MEF2: A MADS box transcription factor expressed in differentiating mesoderm and muscle cell lineages during Drosophila embryogenesis. Proc. Natl. Acad. Sci. 91: 56625666.

Murphy, A.M., T. Lee, C.M. Andrews, B-Z. Shilo, and D.J. Montell. 1995. The breathless FGF receptor homolog, a downstream target of Drosophila C/EBP in the developmental control of cell migration. Development 121: 2255-2263.

Murre, C., P.S. McCaw, and D. Baltimore. 1989. A new DNA binding and dimerization motif in immunoglobulin enhancer binding, daughterless, MyoD and myc proteins. Cell 56: $777-783$.

Nambu, J.R., R.G. Franks, S. Hu, and S.T. Crews. 1990. The single-minded gene of Drosophila is required for the expression of genes important for the development of CNS midline cells. Cell 63: 63-75.

Park, M.Y., X.S. Wu, K. Golden, J.D. Axelrod, and R. Bodmer.
1996. The wingless signaling pathway is directly involved in Drosophila heart development. Dev. Biol. 177: 104-116.

Patel, N.H., P.M. Snow, and C.S. Goodman. 1987. Characterization and cloning of fasciclin III: A glycoprotein expressed on a subset of neurons and axon pathways in Drosophila. Cell 48: 975-988.

Rao, Y., H. Vaessin, L.Y. Jan, and Y.N. Jan. 1991. Neuroectoderm in Drosophila embryos is dependent on the mesoderm for positioning but not for formation. Genes \& Dev. 5: $1577-$ 1588.

Reichman-Fried, M. and B-Z. Shilo. 1995. Breathless, a Drosophila FGF receptor homolog, is required for the onset of tracheal cell migration and tracheole formation. Mech. Dev. 52: $265-273$.

Reichman-Fried, M., B. Dickson, E. Hafen, and B-Z. Shilo. 1994. Elucidation of the role of breathless, a Drosophila FGF receptor homolog, in tracheal cell migration. Genes \& Dev. 8: $428-439$.

Robinson, M.L., L.A. MacMillan-Crow, J.A. Thompson, and P.A. Overbeek. 1995. Expression of a truncated FGF receptor results in defective lens development in transgenic mice. Development 121: 3959-3967.

Shishido, E., S.-I. Higashijima, Y. Emori, and K. Saigo. 1993. Two FGF-receptor homologs of Drosophila: One is expressed in mesodermal primordium in early embryos. Development 117: 751-761.

St. Johnston, D. and C. Nüsslein-Volhard. 1992. The origin of pattern and polarity in the Drosophila embryo. Cell 68: 201219.

Staehling-Hampton, K., F.M. Hoffmann, M.K. Baylies, E. Rushton, and M. Bate. 1994. dpp induces mesodermal gene expression in Drosophila. Nature 372: 783-786.

Thisse, B., C. Stoetzel, T.C. Gorostiza, and S.F. Perrin. 1988. Sequence of the twist gene and nuclear localization of its protein in endomesodermal cells of early Drosophila embryos. EMBO J. 7: 2175-2183.

Volk, T. and K. VijayRaghavan. 1994. A central role for epidermal segment border cells in the induction of muscle patterning in the Drosophila embryo. Development 120: 59-70.

Yamaguchi, T.P., K. Harpal, M. Henkemeyer, and J. Rossant. 1994. fgfr-1 is required for embryonic growth and mesodermal patterning during mouse gastrulation. Genes \& Dev. 8: 3032-3044.

Yarnitzky, T. and T. Volk. 1995. Laminin is required for heart, somatic muscles, and gut development in the Drosophila embryo. Dev. Biol. 169: 609-618.

Zaffran, S., M. Astier, D. Gratecos, A. Guillen, and M. Sémériva. 1995. Cellular interactions during heart morphogenesis in the Drosophila embryo. Biol. Cell 84: 13-24. 


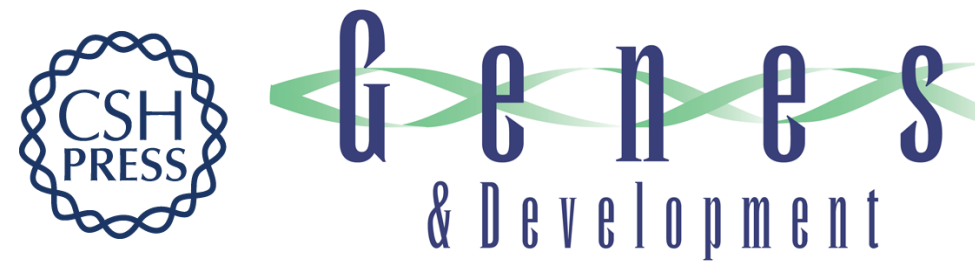

\section{Heartless, a Drosophila FGF receptor homolog, is essential for cell migration and establishment of several mesodermal lineages.}

M Beiman, B Z Shilo and T Volk

Genes Dev. 1996, 10:

Access the most recent version at doi:10.1101/gad.10.23.2993

References This article cites 42 articles, 22 of which can be accessed free at:

http://genesdev.cshlp.org/content/10/23/2993.full.html\#ref-list-1

License

Email Alerting

Service

Receive free email alerts when new articles cite this article - sign up in the box at the top right corner of the article or click here.

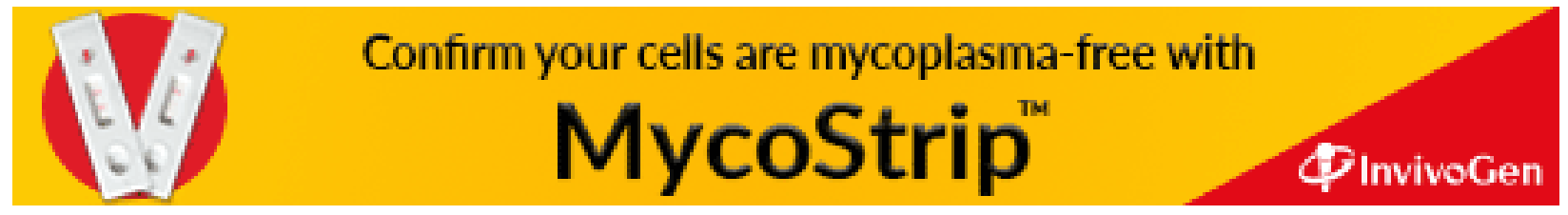

